\title{
Erratum: Shapes of sedimenting soft elastic capsules in a viscous fluid [Phys. Rev. E 92, 033003 (2015)]
}

\author{
Horst-Holger Boltz and Jan Kierfeld \\ (Received 3 December 2015; published 16 December 2015)
}

DOI: 10.1103/PhysRevE.92.069904

PACS number(s): 47.57.ef, 83.10.-y, 46.70.De, 46.32.+x, 99.10.Cd

We want to point out errors that occurred in the calculation regarding the rotational stability of sedimenting capsule shapes in Eq. (D4) in Appendix D and in the stability criterion used in Sec. III E resulting in an incorrect Fig. 8.

The gravitational torque in Eq. (D4) should read

$$
\mathbf{e}_{y} \cdot \mathbf{T}_{g}^{\prime}=\pi d \alpha g \int d s \sin \psi r^{2}\left(z-z_{0}\right) .
$$

Equating this gravitational torque (D4) with the hydrodynamic torque (D3) determines the pivot point $z_{0}$ of the rotation. We also want to point out that this pivot point $z_{0}$, which we called the center of hydrodynamic stress, actually differs from the center of hydrodynamic stress as defined in Ref. [1], where it is the point $z_{0}$ where the hydrodynamic torque (D3) itself equals zero. The shape is stable with respect to rotations if the pivot point $z_{0}$ (or the center of hydrodynamic stress according to Ref. [1]) lies above the center of gravity $z_{\mathrm{c} . \mathrm{m} \text {. }}$ Using this corrected criterion for rotational stability and the corrected gravitational torque (D4), we obtain a corrected version of Fig. 8, which is shown as Fig. 1.

Most of our results are qualitatively unchanged: We find that both of the buckled sedimenting capsule shapes are stable, the pseudospherical shape is only weakly instable for small gravitational forces, and the pear shape is stable for high gravitational forces. Pseudospherical and pear shape develop a pronounced instability only for gravity forces $g$ close to the crossover or transition from pseudospherical to pear shapes, as shown in Fig. 1.

Moreover, in the text following Eq. (18) the correct equations for tensions and bending moments should read $\tau_{s}=\lambda_{\varphi}^{-1} \partial w_{s} / \partial e_{s}$ and $m_{s}=\lambda_{\varphi}^{-1} \partial w_{s} / \partial K_{s}$.

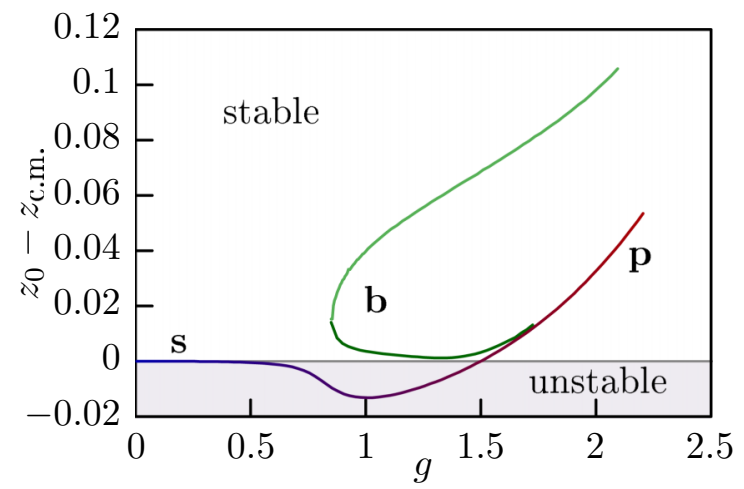

FIG. 1. (Color online) Linear analysis concerning rotational stability for $\tilde{E}_{B}=0.01$. We show the (dimensionless) difference $z_{0}-z_{\mathrm{c} . \mathrm{m}}$.

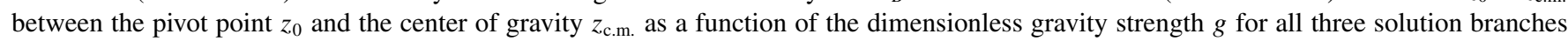
[pseudospherical (blue), pear-shaped (red), and buckled (green)]. For $z_{0}-z_{\text {c.m. }}>0$, i.e., above the gray line, the shape is linearly stable against out-of-axis rotations. Only solutions for gravity force $g$ close to the crossover or transition from pseudospherical to pear shapes develop a pronounced instability.

[1] J. Happel and H. Brenner, Low Reynolds Number Hydrodynamics: With Special Applications to Particulate Media (Springer, Berlin, 1983), Vol. 1. 